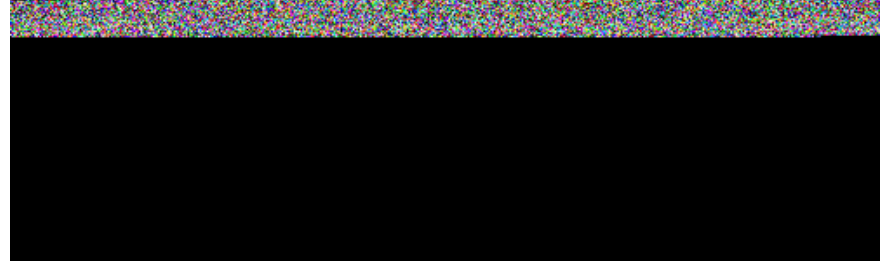

This information is current as of April 26, 2023.

\title{
Brain Perfusion Measurements Using Multidelay Arterial Spin-Labeling Are Systematically Biased by the Number of Delays
}

M. van der Thiel, C. Rodriguez, P. Giannakopoulos, M.X. Burke, R. Marc Lebel, N. Gninenko, D. Van De Ville and S. Haller

AJNR Am J Neuroradiol 2018, 39 (8) 1432-1438 doi: https://doi.org/10.3174/ajnr.A5717 http://www.ajnr.org/content/39/8/1432 


\title{
Brain Perfusion Measurements Using Multidelay Arterial Spin-Labeling Are Systematically Biased by the Number of Delays
}

(D) M. van der Thiel, (D) Rodriguez, (DP. Giannakopoulos, (D).X. Burke, DR. Marc Lebel, (D) N. Gninenko, (DD. Van De Ville, and (D) S. Haller

\begin{abstract}
BACKGROUND AND PURPOSE: Multidelay arterial spin-labeling is a promising emerging method in clinical practice. The effect of imaging parameters in multidelay arterial spin-labeling on estimated cerebral blood flow measurements remains unknown. We directly compared 3-delay versus 7-delay sequences, assessing the difference in the estimated transit time and blood flow.
\end{abstract}

MATERIALS AND METHODS: This study included 87 cognitively healthy controls ( $78.7 \pm 3.8$ years of age; 49 women). We assessed delay and transit time-uncorrected and transit time-corrected CBF maps. Data analysis included voxelwise permutation-based betweensequence comparisons of 3-delay versus 7-delay, within-sequence comparison of transit time-uncorrected versus transit time-corrected maps, and average CBF calculations in regions that have been shown to differ.

RESULTS: The 7-delay sequence estimated a higher CBF value than the 3-delay for the transit time-uncorrected and transit timecorrected maps in regions corresponding to the watershed areas (transit time-uncorrected $=27.62 \pm 12.23$ versus $24.58 \pm 11.70 \mathrm{~mL} / \mathrm{min} /$ $100 \mathrm{~g}$, Cohen's $d=0.25$; transit time-corrected $=33.48 \pm 14.92$ versus $30.16 \pm 14.32 \mathrm{~mL} / \mathrm{min} / 100 \mathrm{~g}$, Cohen's $d=0.23)$. In the peripheral regions of the brain, the estimated delay was found to be longer for the 3 -delay sequence $(1.52408 \pm 0.25236$ seconds versus $1.47755 \pm$ 0.24242 seconds, Cohen's $d=0.19)$, while the inverse was found in the center of the brain $(1.39388 \pm 0.22056$ seconds versus $1.42565 \pm$ 0.21872 seconds, Cohen's $d=0.14$ ). Moreover, 7-delay had lower hemispheric asymmetry.

CONCLUSIONS: The results of this study support the necessity of standardizing acquisition parameters in multidelay arterial spin-labeling and identifying basic parameters as a confounding factor in CBF quantification studies. Our findings conclude that multidelay arterial spin-labeling sequences with a high number of delays estimate higher CBF values than those with a lower number of delays.

ABBREVIATIONS: ASL = arterial spin-labeling; $d=$ Cohen's $d ;$ PLD $=$ postlabeling delay

A rterial spin-labeling (ASL) is a noninvasive MR imaging technique capable of measuring perfusion and quantifying cerebral blood flow. ASL uses water in the blood as an endogenous

Received November 16, 2017; accepted after revision May 9, 2018.

From the Faculty of Medicine of the University of Geneva (M.v.d.T., P.G., N.G. D.v.d.V., S.H.), Geneva, Switzerland; Institute of Bioengineering (M.v.d.T., N.G., D.v.d.V.), School of Engineering, École Polytechnique Fédérale de Lausanne, Lausanne, Switzerland; Division of Institutional Measures (C.R., P.G.), Medical Direction, University Hospitals of Geneva, Geneva, Switzerland; Affidea Centre de Diagnostic Radiologique de Carouge (S.H.), Geneva, Switzerland; Department of Surgical Sciences and Radiology (S.H.), Uppsala University, Uppsala, Sweden; and GE Healthcare (M.X.B., M.L.), Little Chalfont, UK.

This work was supported by Swiss National Foundation grants SNF 3200B0-1161193 and SPUM 33CM30-124111 and an unrestricted grant from the Association Suisse pour la Recherche Alzheimer.

Please address correspondence to Sven Haller, MD, Faculty of Medicine of the University of Geneva, Switzerland, Affidea Centre de Diagnostic Radiologique de Carouge, Geneva, Switzerland, Department of Surgical Sciences and Radiology, Uppsala University, Uppsala, Sweden; e-mail: sven.haller@gmail.com

- Indicates open access to non-subscribers at www.ajnr.org

http://dx.doi.org/10.3174/ajnr.A5717 tracer by inversion of proximal inflowing spins, ${ }^{1,2}$ which are exchanged with tissue water, giving rise to the perfusion signal in the inversion images. ${ }^{3}$ The arterial transit time is reflective of the time it takes for blood to travel from the labeling to imaging plane. The time between labeling and acquisition is called the postlabeling delay (PLD). ${ }^{4}$ The use of different PLDs is implemented in ASL to account for the arterial transit time. ${ }^{5}$ The measurement of the cerebral blood flow without incorporating the estimated PLD gives rise to the transit time- uncorrected maps, while the transit time-corrected maps refer to the measurement of CBF corrected for the estimated PLD. Not considering the arterial transit time can lead to underestimation of the CBF signal, ${ }^{6}$ indicating the importance of correction for the transit time.

In practice, the arterial transit time is found to be a considerable source of error in the evaluation of perfusion. ${ }^{3,7}$ Customarily, ASL measurements are administered using a single PLD. ${ }^{8}$ However, when the distribution of transit times is wide, as observed in cerebrovascular diseases with multiple arterial lesions and proxi- 


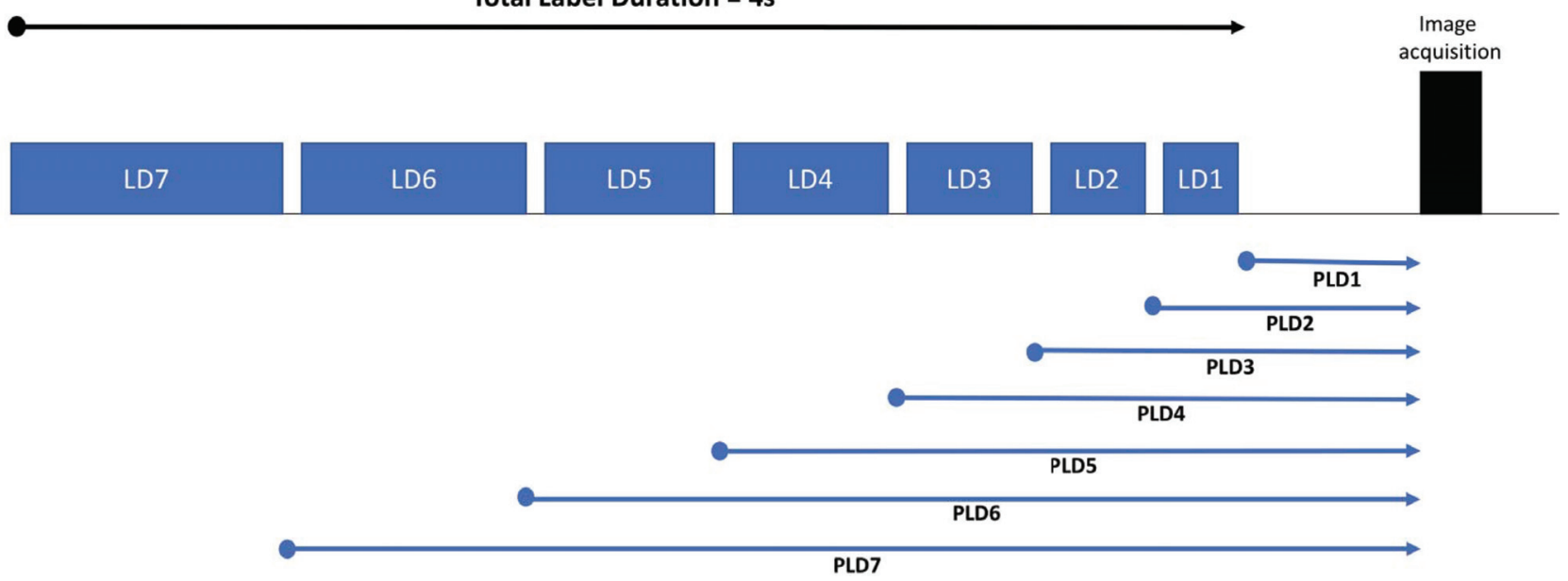

FIG 1. Seven pulsed continuous arterial spin-labeling periods of different durations: labeling duration $(\mathrm{LD})=0.22,0.26,0.30,0.37,0.48,0.68$, and 1.18 seconds was used to acquire perfusion-weighted images at postlabeling delays of 1.00, 1.22, 1.48, 1.78, 2.15, 2.62, and 3.32 seconds. When we used only 3 labeling periods, $L D=0.57,0.89,2.04$ seconds and PLD $=1.00,1.57$, and 2.46 seconds.

mal vascular stenosis (a frequent finding in older subjects ${ }^{6,9}$ ), these methods will be imprecise. One of the difficulties regarding the PLD is the modification of the optimal PLD during the life span. In general, children and young adults have good cardiac output and consequently a short optimal PLD. During aging, cardiac output decreases and vascular comorbidity increases, so a longer PLD is recommended for elderly participants. ${ }^{10}$ The PLD being too short may lead to a false underestimation of the CBF.

This problem can be partially solved by acquiring images at multiple PLDs, thereby sampling several points along the ASL uptake and decay curve. ${ }^{4}$ Multidelay ASL using a Hadamard-encoding scheme generates ASL images with several postlabeling delay times and enables quantification of both perfusion and arterial transit time. ${ }^{11}$ In addition, the increased data averaging within the Hadamard-encoding scheme results in a higher SNR and reduces physiologic background noise. ${ }^{11}$ Furthermore, knowledge of the transit time permits more robust CBF quantification than methods naive to the arrival time. Multiple PLDs are expected to improve the accuracy of CBF quantification ${ }^{12}$ but sacrifice SNR efficiency due to short label durations. ${ }^{9}$ Conversely, a sequence with fewer delays will have a higher SNR per time point compared with many delays; nevertheless, it might sacrifice the specificity of the delay estimates, leading to a less accurate correction for this delay. The appropriate number of delay times to balance accurate transit-time estimates and precise CBF quantification is unclear.

The current study will examine the impact of the number of delays and transit-time correction on the estimation of CBF in ASL. The results of 3-delay and 7-delay sequences will be compared, and the effect of the transit-time correction on 3-delay and 7-delay sequences will be considered independently.

\section{MATERIALS AND METHODS \\ Participants}

The data used in this article were retrieved from an ongoing large population-based longitudinal study. The study encompassed a comprehensive neuropsychological evaluation, as described in detail previously. ${ }^{13}$ The final sample included 87 participants, classified as cognitively healthy controls (mean age, $78.7 \pm 3.8$ years; 49 women). All participants gave written informed consent after formal approval by the local ethics committee.

\section{MR Imaging}

Imaging was performed on a 3T MR750w scanner (GE Healthcare, Milwaukee, Wisconsin) using a 32-channel head array coil. Perfusion images were acquired with a background-suppressed 3D stack-of-spirals FSE sequence preceded by a Hadamard-encoded pseudocontinuous arterial spin-labeling module (modified to acquire an additional control-only phase). A total label duration of 4 seconds was encoded into either 3 or 7 sub-blocks (Fig 1). The label durations of the 7 labeling blocks were $0.22,0.26,0.30$, $0.37,0.48,0.68$, and 1.18 seconds; postlabeling delays were 1.00 , $1.22,1.48,1.78,2.15,2.62$, and 3.32 seconds.

The total scan time was 4.02 minutes. When we used only 3 labeling blocks, label durations were $0.57,0.89$, and 2.04 seconds and postlabeling delays were $1.00,1.57$, and 2.46 seconds, resulting in a total scan time of 3.37 minutes.

Key imaging parameters were the following: $\mathrm{FOV}=22.0 \mathrm{~cm}$, slice thickness $=4.0 \mathrm{~mm}, 32$ slices, bandwidth $= \pm 62.5 \mathrm{kHz}, 4$ arms with 640 points each. Images with 3 labeling blocks were acquired with a TE of 0.0105 seconds and a TR of 5.978 seconds; and for the 7 labeling blocks, these were $0.0105 \mathrm{~ms}$ and 5.936 seconds, respectively. Acquisition included a proton-densityweighted reference image with matched parameters for quantification. Image reconstruction was performed using an IDL-based RECON code, and reconstructed images were stored as DICOM images in the data base of the scanner.

Images were created at all delay times. The combined delay map consisted of the sum of the delay times per subject. In addition, a transit-delay map reflecting the transit time of blood from the labeling plane to the imaging plane was estimated using the signal-weighted delay method described by Dai et al. ${ }^{14}$ Transit time-uncorrected CBF was computed using the combined delay image. Transit time-corrected CBF maps were calculated by incorporating the combined delay image and the transit delay $(\delta)$ : 


$$
C B F=\frac{6000 e^{\delta / T_{1 a}}}{2 \varepsilon T_{1 a}\left(e^{-\max (P L D-\delta, 0) / T_{1 t}}-e^{-\max (L D+P L D-\delta, 0) / T_{1 t}}\right)} \frac{P}{\frac{R}{S_{r}}},
$$

where CBF reflects the cerebral blood flow in milliliters/100 $\mathrm{g} / \mathrm{min}, \mathrm{PLD}$ is the shortest postlabeling delay (1.0 seconds), LD is the entire labeling duration (4.0 seconds), $T_{1 \mathrm{a}}$ is the longitudinal relaxation of arterial blood (1.6 seconds), $T_{1 \mathrm{t}}$ is the longitudinal relaxation of gray matter ( 1.2 seconds), $\varepsilon$ is the combined efficiency of labeling and background suppression (0.6375), $P$ is the signal intensity in the perfusion-weighted image, and $R$ is the signal intensity of the reference image.

The reference image is scaled by $s_{\mathrm{r}}=\lambda\left(1-e^{-2 / 1.2}\right)$ to account for the combined effect of the blood-brain partition coefficient (lambda $=0.9$ ) and the partial GM signal recovery in a 2 -second saturation-recovery reference image. The in-plane resolution of the resulting images was $1.88 \times 1.88 \times 4 \mathrm{~mm}$.

\section{Statistical Analysis}

Data Preprocessing. The ASL data were processed using the fMRIB Software Library (FSL, Version 5.0.9; http://fsl.fmrib.ox.ac.uk/fsl). The combined delay image was obtained per participant, and nonbrain tissue was removed using the FSL Brain Extraction Tool (http://fsl.fmrib.ox.ac.uk/fsl/fslwiki/BET). The brain-extracted combined delay maps were normalized to the Montreal Neurological Institute standard space using an echoplanar image template from the Statistical Parametric Mapping SPM8 (http://www.fil.ion.ucl.ac.uk/spm/software/spm12) toolbox standard space using linear registration (FMRIB Linear Image Registration Tool; http://www.fmrib.ox.ac.uk/fslwiki/FLIRT, part of FSL). The concatenated transformation matrix of the transit-delay maps was then applied to the uncorrected flow maps, the transit time-corrected flow maps, and the transit-delay maps to spatially normalize the data to the EPI template.

The normalized ASL images were smoothed with a $5-\mathrm{mm}$ full width at half maximum Gaussian kernel using a dilated 2-mm brainextracted Montreal Neurological Institute mask (FSLUTILS; ftp:// surfer.nmr.mgh.harvard.edu/pub/dist/freesurfer/tutorial_packages/ OSX/fsl_501/doc/wiki/Fslutils.html, part of FSL).

\section{3-Delay versus 7-Delay}

First, the 7-delay and 3-delay sequences were compared using a voxelwise permutation-based testing (Randomise; http://fsl.fmrib. ox.ac.uk/fsl/fslwiki/Randomise, part of FSL), with threshold-free cluster enhancement correction for multiple comparisons applied $^{15}$ and $P<.05$ considered significant.

Five thousand permutations were executed per contrast. The dilated Montreal Neurological Institute 2-mm brain-extracted mask was used during randomization for masking of nonbrain voxels. A repeated measures design was administered with age and sex as nonexplanatory coregressors. ${ }^{16}$ Age was defined as the difference in days between the date of birth and the day of scanning.

Second, the voxels that showed either a positive or negative significant difference between the 7-delay and 3-delay signal were masked by a threshold of 0.95 per direction of the difference (FSLUTILS). The mean values of all participants combined for the 7-delay and the 3-delay within the resulting masks were cal- culated (FSLUTILS). The Cohen d was determined to imply the effect size of the discrepancy between sequences.

\section{Corrected-versus-Uncorrected Flow Maps}

The preprocessed transit-uncorrected and transit-corrected flow images of the 7-delay sequence and, independently, of the 3-delay sequence were likewise compared using voxelwise permutationbased testing (Randomise). The mean values of all participants within the resulting masks of the uncorrected and the corrected maps were calculated separately (FSLUTILS).

\section{Laterality Index}

The laterality index is a way to indicate hemispheric dominance. With the expectation of a symmetric perfusion distribution within the brain, an unbiased estimate of this would result in a laterality index of approximately zero. ${ }^{17}$ The laterality index for the transit time-corrected flow maps was determined for the 7 -delay sequences and the 3-delay sequences. The right and left hemispheres were masked separately, and the mean CBF value per hemisphere was calculated within participants. The laterality index (LI) was determined as described in Seghier ${ }^{17}$ :

$$
L I=\frac{Q_{\mathrm{LH}}-Q_{\mathrm{RH}}}{Q_{\mathrm{LH}}+Q_{\mathrm{RH}}}
$$

where $Q_{\mathrm{LH}}$ and $Q_{\mathrm{RH}}$ are representative quantities for the left and right hemisphere contributions of $\mathrm{CBF}$, respectively. A positive laterality index will represent a left-lateralization effect, and a negative value stands for right-hemisphere dominance. A paired $t$ test was applied to compare the laterality indices of the 2 sequences.

\section{RESULTS}

\section{Voxelwise Comparison between 3-Delay and 7-Delay}

Transit Time-Uncorrected Flow Maps. The 7-delay sequence provided a higher estimation of $\mathrm{CBF}$ in the peripheral brain regions and the center of the brain and regions between them compared with the 3-delay sequence. Most interesting, this difference was profound in the watershed areas of the brain (Fig 2A). ${ }^{18}$ Within the voxels that had a significant difference, the 7-delay sequences estimated an $11 \%$ higher average CBF value compared with the 3-delay estimates $(27.62 \pm 12.23$ versus $24.58 \pm 11.70$ $\mathrm{mL} / \mathrm{min} / 100 \mathrm{~g}, 11.00 \%$ difference, Cohen's $d=0.25)($ Fig $2 B)$.

The voxelwise comparison of the transit time-uncorrected flow maps of the 3-delay with the 7-delay established a 7-delay $\mathrm{CBF}$ with an estimate of half of the $\mathrm{CBF}$ value of the estimate of the 3 -delay estimate within the right insular region of the brain $(19.20 \pm 21.29$ versus $12.57 \pm 18.66 \mathrm{~mL} / \mathrm{min} / 100 \mathrm{~g}, 52.70 \%$ difference, $d=0.33)$.

Transit Time-Corrected Flow Maps. The average CBF estimates of the 7-delay transit-corrected flow maps were found to be significantly higher than the 3-delay estimates in the regions corresponding approximately to the results of the transit-uncorrected flow maps (Fig 2A). For the uncorrected maps, the discrepancy between the 7-delay and 3-delay was found to be more global, but lower estimated CBF values were determined within these voxels, as illustrated in Fig $2 B(33.48 \pm 14.92$ versus $30.16 \pm 14.32 \mathrm{~mL} /$ $\mathrm{min} / 100 \mathrm{~g}, 9.91 \%$ difference, $\mathrm{d}=0.23$ ). The voxelwise compari- 


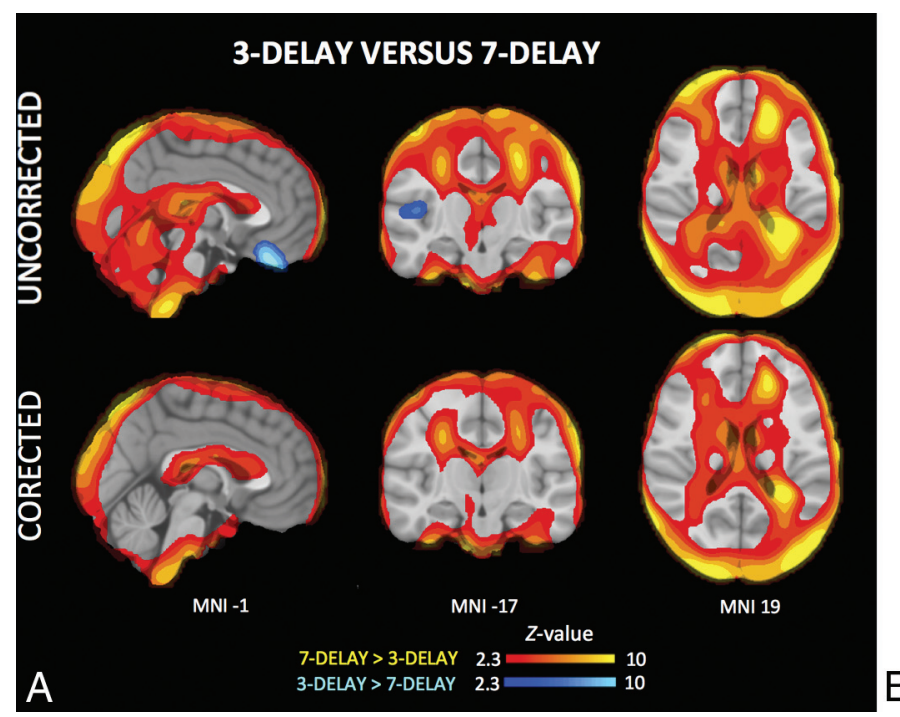

\section{AVERAGE ESTIMATED FLOW HIGHER ESTIMATE FOR 7-DELAY}

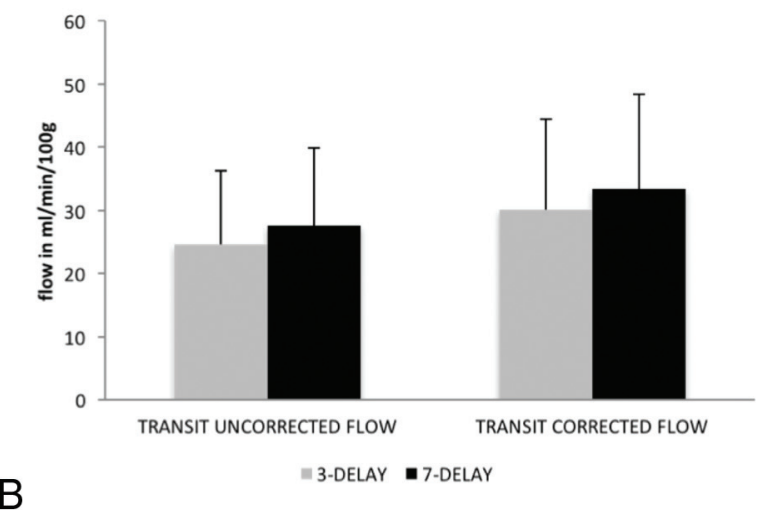

FIG 2. A, The spatial distribution of the differences between the 7-delay sequence and the 3-delay sequence for the uncorrected and corrected flow maps. The higher estimation value for the 7-delay over the 3-delay sequences is presented in the areas corresponding to the watershed areas (yellow). The 7-delay CBF estimates were found to be half the value of the 3-delay transit time-corrected flow maps within the right insular region of the brain and frontal regions (blue). The transit time-corrected maps show a higher value for the 7-delay over the 3-delay sequence in the regions overlapping the uncorrected maps. However, the transit time-uncorrected maps show a broader signal for the difference between the 2 sequences, including more posterior regions of the brain. $B$, The average estimated transit-uncorrected flow and the transitcorrected flow in milliliters/minute/100 g for the 7-delay and 3-delay sequences within the voxels that displayed a significantly higher estimate for the 7-delay sequences. For these voxels, an average perfusion value of $27.62 \pm 12.23$ is estimated by the 7-delay versus $24.58 \pm 11.70$ $\mathrm{mL} / \mathrm{min} / 100 \mathrm{~g}$ for the 3-delay sequences in the uncorrected maps, and a perfusion value of $33.48 \pm 14.92$ is estimated by the 7-delay versus $30.16 \pm 14.32 \mathrm{~mL} / \mathrm{min} / 100 \mathrm{~g}$ for the 3-delay sequences of the corrected flow maps.
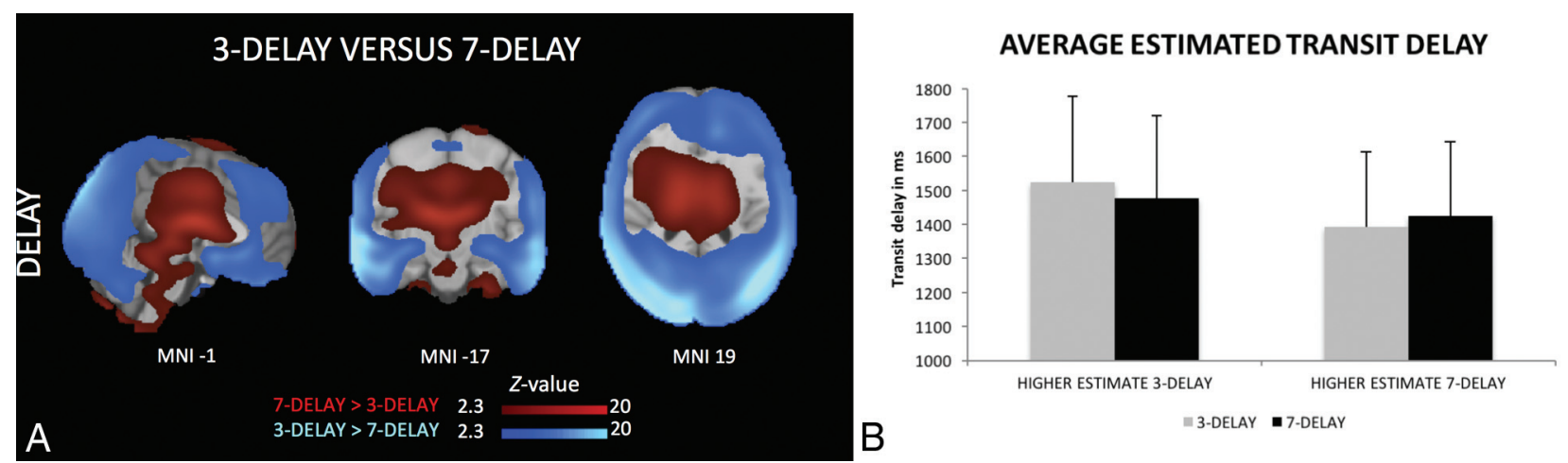

FIG 3. A, The spatial distribution of the differences between the 7-delay sequence and the 3-delay sequence for the delay maps. The estimation of the 7-delay as inferior to the 3-delay sequence (blue) concerns peripheral areas of the brain. In contrast, within the central regions of the brain, the averaged transit-time estimates are longer for the 7-delay sequences compared with the 3-delay sequences (red). B, The average estimated transit time delay in milliseconds of the voxels that displayed a significantly higher estimate for the 7-delay and those that showed a higher estimate for the 3-delay, respectively. This figure shows the difference between the 7-delay and the 3-delay within these corresponding regions. For the regions where the 7-delay estimated a smaller arterial transit time delay than the 3-delay sequence, an average arterial transit time of $1.47755 \pm 0.24242$ seconds is found for the 7-delay versus $1.52408 \pm 0.25236$ seconds for the 3 -delay. Regions with a longer arterial transit time delay for the 7-delay compared to 3-delay resulted in an average arterial transit time estimation of $1.42565 \pm 0.21872$ seconds by the 7-delay sequence and $1.39388 \pm 0.22056$ seconds by the 3-delay sequences for the transit-time delay.

son of the corrected CBF maps testing for lower estimates for the 7-delay sequences compared with the 3-delay sequences was found to be statistically nonsignificant within the whole brain.

Transit-Delay Maps. In the peripheral regions of the brain, the transit-time estimates were found to be significantly shorter for the 7-delay sequence compared with the 3-delay sequence (Fig $3 A$ ). The mean difference between the delay sequences within the significant voxels corresponds to a medium effect size (1.47755 \pm 0.24242 seconds versus $1.52408 \pm 0.25236$ seconds, $3.15 \%$ difference, $d=0.19$ ) (Fig 3B).

Most interesting, in the center of the brain (Fig 3A), the 7-de- lay sequence led to a significantly longer estimated transit time than the 3 -delay measurements $(1.42565 \pm 0.21872$ seconds versus $1.39388 \pm 0.22056$ seconds, $2.23 \%$ difference, $d=0.14$ ) (Fig 3B).

\section{Voxelwise Comparison between Transit-Corrected and-Uncorrected Maps}

On average across the entire brain, the transit time-corrected flow maps provided an estimation of almost $20 \%$, exceeding the CBF value of the corresponding uncorrected flow maps, for both the 7-delay and 3-delay sequences (7-delay: $34.56 \pm 13.58$ versus $28.60 \pm$ 


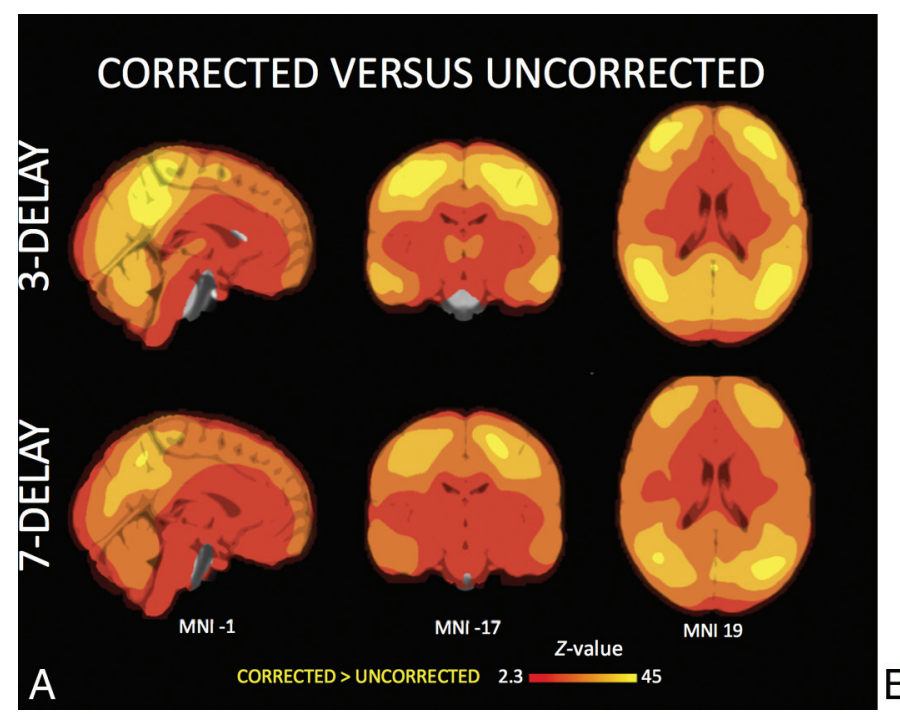

\section{AVERAGE CORRECTED AND UNCORRECTED FLOW}

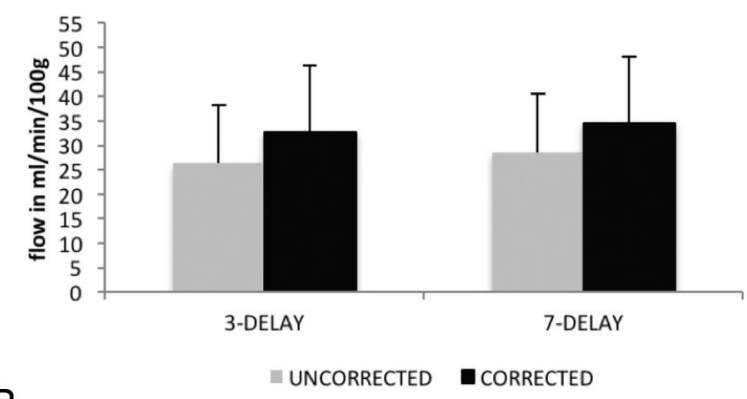

FIG 4. A, The spatial distribution of the differences between the uncorrected and corrected flow maps for the 7-delay and 3-delay sequences. On average, the transit-corrected flow maps provided a higher value for both the 7-delay and 3-delay compared with the corresponding uncorrected flow maps across the entire brain. $B$, The average estimated transit-uncorrected flow and the transit-corrected flow in milliliters/ minute/100 $\mathrm{g}$ within the voxels that showed a significantly higher estimate for the transit-corrected sequences than for the 7-delay and the 3-delay sequences. This figure shows the difference between the uncorrected and corrected maps. The 7-delay sequences estimated a mean perfusion value of $28.60 \pm 11.91 \mathrm{~mL} / \mathrm{min} / 100 \mathrm{~g}$ for the uncorrected versus $34.56 \pm 13.58 \mathrm{~mL} / \mathrm{min} / 100 \mathrm{~g}$ for the corrected flow maps. For the 3-delay sequences, the uncorrected flow map resulted in an average perfusion estimation of $26.38 \pm 11.74 \mathrm{~mL} / \mathrm{min} / 100 \mathrm{~g}$ versus the corrected map of $32.78 \pm 13.44 \mathrm{~mL} / \mathrm{min} / 100 \mathrm{~g}$. The corrected flow maps estimate for both sequences almost an additional $20 \%$ of the perfusion value of the uncorrected flow maps.

$11.91 \mathrm{~mL} / \mathrm{min} / 100 \mathrm{~g}, 17.25 \%$ difference, $d=0.47 ; 3$-delay: $32.78 \pm$ 13.44 versus $26.38 \pm 11.74 \mathrm{~mL} / \mathrm{min} / 100 \mathrm{~g}, 19.52 \%$ difference, $\mathrm{d}=$ 0.53 ) (Fig 4).

Laterality Index. The 7-delay and 3-delay sequences both represented a higher CBF signal in the right hemisphere. A more symmetric pattern was found for the 7-delay (mean $=-0.013 \pm$ $0.035)$ compared with the greater average hemispheric asymmetry of the 3-delay sequence of $2.3 \%$ (mean $=-0.023 \pm 0.35$ ). The right lateralization was established as significantly lower in the 7-delay sequence compared with the 3 -delay sequence $(t$-value $=$ 3.89, $P<.001)$.

\section{DISCUSSION}

The current study looked at 3-delay and 7-delay sequences by comparing the corrected, uncorrected, and delay maps. A greater CBF signal was found for both the uncorrected and corrected flow maps of the 7-delay sequence compared with the 3-delay sequence. These findings led us to conclude that multidelay ASL sequences with high numbers of delays lead to an estimation of higher CBF values than those with lower numbers of delays.

\section{Comparison of 3-Delay versus 7-Delay on the Corrected and Uncorrected Flow Maps}

Most interesting, the 7-delay surpassed the 3-delay in both the transit-uncorrected and transit-corrected flow maps in regions relating to the borderzone or watershed areas of the brain. The watershed areas are at distal parts of each vascular territory and therefore have a longer and more variable arterial transit time than other portions of each vascular territory. ${ }^{18}$ In consequence, estimation of the arterial transit time within these regions tends to be more challenging. A delay sequence with a higher number of delays solves this problem by acquiring images at multiple PLD times, thereby measuring the entire delay curve more precisely. ${ }^{4}$ The advantages of a multidelay ASL sequence with a higher number of delays especially is seen when the distribution of transit times is wide, like that observed in older populations, as well as in some pathologies and cerebrovascular diseases with multiple arterial lesions. ${ }^{6,9}$ The elderly sample used in the current study would be sensitive to the effects of the advantage of a sequence with a higher number of PLDs, highlighting the effects of PLD choice on the estimated CBF signal within the watershed areas.

However, the precision of arterial transit time estimation by a sequence with more delay could be at the expense of the signalto-noise ratio per unit of time. ${ }^{5}$ In line with these statements, the current study determined a lower estimation of the 7-delay sequence than the 3-delay, within the right insular region of the brain in the transit time-corrected flow maps. The insular region is the center of the vascularization and is one of the first regions to be vascularized. ${ }^{19}$ Thus, these areas will benefit less from the advantage in the estimation of longer arterial transit times of the sequence with a high number of delays. The CBF within the insular region might be underestimated by the 7-delay sequence, whereas the watershed areas benefit by obtaining a higher CBF signal.

Most interesting, this lower estimation of the 7-delay as opposed to the 3-delay is not observed within the uncorrected flow maps. A possible conclusion would be that correction for the transit time increases the overestimation of the 3-delay sequence within the right insular region.

In addition to the effects of the chosen number of delays, the current study showed the impact of the combination of different multidelays and correction for arterial transit time. We found a 
larger increase in the estimated CBF value after correction for arterial transit time with the 7-delay sequence than with the 3-delay sequence. These findings suggest a stronger effect of transittime correction on the delay sequence with a higher number of delays. Not only is the collection of parameters for the multidelay ASL important, but also the choice of combining different parameters can further influence CBF results.

\section{Comparison of 3-Delay versus 7-Delay on the Transit-Delay Maps}

The 3-delay and 7-delay sequences were further compared by looking at the transit-delay maps. The 7-delay demonstrated a shorter arterial transit time in the peripheral region sequence and a longer transit time in the central regions of the brain compared with the 3-delay. Most interesting, the central regions of the brain where this effect is apparent receive their blood supply mainly from the middle cerebral artery, ${ }^{18}$ suggesting an effect of the vascularization on these findings.

In addition, with a short PLD time, arterial signal is likely to artificially decrease the estimated transit delay in regions with substantial contamination. ${ }^{11}$ This decrease in estimated transit delay will propagate into low transit-corrected CBF values. Our results show a shorter transit delay in the central regions of the brain with the 3-delay protocol, which are more likely to be contaminated by arterial signal. Additionally, regions like the insular cortex may be prone to arterial contamination, which could also explain why the 3-delay protocol displays greater CBF than the 7-delay protocol in this area. We chose not to use vascular crushing in this study. The risk with using vascular crushing is a substantially reduced $\mathrm{SNR},{ }^{3}$ enhanced sensitivity to motion, and possible removal of important clinical information. ${ }^{11}$

Another point of influence might be the distribution of scans into the different time bins in the multidelay sequences. In a lower number of delays, more averages can be taken per each time bin, resulting in a higher SNR for each bin. This comes at the cost of having fewer time points. Therefore, time-related measures such as the delay maps are estimated less accurately. Not only are there fewer time bins, but also each time bin is longer; this feature results in a lower accuracy for estimating time-derived parameters for the 3-delay versus the 7-delay protocol.

The observed local differences in the delay maps of the 3-delay and the 7-delay sequences could be explained by this difference in time bins.

\section{Comparison of 3-Delay versus 7-Delay in the Laterality Index}

Both the 7-delay and 3-delay sequences represented a higher CBF signal in the right hemisphere compared with the left hemisphere; nevertheless, the mean laterality index determined within the 3-delay sequence was found to be significantly greater than the asymmetry of the 7-delay. This asymmetry of the CBF measures could have possibly led to the asymmetric findings within the corrected flow maps regarding the right insular region.

With the expectation of a symmetric perfusion distribution within the brain, one might argue that the 7-delay sequence provides a less biased global CBF approximation in comparison with the more asymmetric measurement of the 3-delay sequence.
However, the observed difference is relatively small (2\%), and multiple other explanations could be argued, such as the acclimatization of the patient to the MR imaging environment rather than the sequence. Although there is an obvious explanation for these findings, it could be of interest to study the effects of number of delays on hemispherical differences in CBF estimation in more detail in future studies.

\section{Comparison of the Transit-Corrected versus -Uncorrected Flow Maps}

The current study investigated the effect of transit-time correction by comparing the transit-corrected flow maps with the transit-uncorrected flow maps. We established a higher average estimated CBF value for the corrected flow maps than for the uncorrected flow maps within the whole brain. This effect was constant in both the 7-delay and 3-delay sequences.

Although there was no regional variation found within the brain, the correction for delayed flow evidently influences the CBF signal measured. ${ }^{8}$ Hence, this study highlights the importance of applying arterial transit time correction.

The current study identified a 10\% difference in CBF estimates between the 3-delay and 7-delay sequence. In clinical practice, where the differences between healthy subjects and patients are usually of a lower value than this $10 \%$, the chosen number of delays in multidelay ASL could have an influential impact on the diagnostic process. In other respects, the quantification of $\mathrm{CBF}$ values could become biased by the mixed use of the different multidelay ASL sequences.

\section{Limitations}

The current study investigated a 7-delay and a 3-delay sequence to validate the effect of transit-time correction. Future research is recommended to access numerous different multidelay ASL sequences to confirm our conclusions and to find the optimal PLD for a specific region or research objective.

In addition, only the single ASL protocol was applied in this study. To further generalize the findings, one should investigate the scanners from distinct venders and assess protocols variant to pulsed continuous arterial spin-labeling. Moreover, we acknowledge that the dynamic range of transit-delay mapping differs between the 3- and 7-delay protocols in the current study. The alternative (using equally spaced delays) provides images with diminishing SNR and increasing PLD.

A recent work by Guo et $\mathrm{al}^{20}$ compared 3- and 7-delay ASL with linear and exponential delay spacing. They demonstrated that linear spacing outperformed exponential spacing. Their results are consistent with our findings when the relative SNR is greater than 2: Seven-delays provide a higher transit time delay estimate and higher CBF than 3-delays. Further work should focus on optimizing the minimum PLD, the delay spacing, and the use of vascular crushers.

Moreover, future studies are recommended to investigate differences between a low- and high-number multidelay sequence by investigating a sample with subjects of various ages. It is important to consider the relatively older sample size used to determine the advantage of a higher number delay sequence in this study. Nevertheless, for the investigation of elderly and pathologic pop- 
ulations, as is the custom in clinical practice, the 7-delay sequence seems to be strongly preferable to the 3-delay sequence.

\section{CONCLUSIONS}

The results of this study support the necessity of standardizing acquisition parameters in multidelay ASL and identifying basic parameters as a confounding factor in CBF quantification studies. Additionally, the importance of applying arterial transit time correction was highlighted.

Disclosures: Merel van der Thiel—RELATED: Grant: This work is supported by Swiss National Foundation grants SNF 3200B0-1161193 and SPUM 33CM30-124111 and an unrestricted grant from the Association Suisse pour la Recherche Alzheimer.* Michael X. Burke-UNRELATED: Employment: GE Healthcare, Comments: full-time employee. R. Marc Lebel_UNRELATED: Employment: GE Healthcare; Stock/Stock Options: GE Healthcare, Comments: R.M.L. is a shareholder in GE. *Money paid to institution.

\section{REFERENCES}

1. Detre JA, Leigh JS, Williams DS, et al. Perfusion imaging. Magn Reson Med 1992;23:37-45 CrossRef Medline

2. Williams DS, Detre JA, Leigh JS, et al. Magnetic resonance imaging of perfusion using spin inversion of arterial water. Proc Natl Acad Sci U S A 1992;89:212-16 CrossRef Medline

3. Petcharunpaisan S, Ramalho J, Castillo M. Arterial spin labeling in neuroimaging. World J Radiol 2010;2:384-98 CrossRef Medline

4. Petersen ET, Lim T, Golay X. Model-free arterial spin labeling quantification approach for perfusion MRI. Magn Reson Med 2006;55: 219-32 CrossRef Medline

5. Detre JA, Alsop DC. Perfusion magnetic resonance imaging with continuous arterial spin labeling: methods and clinical applications in the central nervous system. Eur J Radiol 1999;30:115-24 CrossRef Medline

6. Ferré JC, Bannier E, Raoult H, et al. Arterial spin labeling (ASL) perfusion: techniques and clinical use. Diagn Interv Imaging 2013; 94:1211-23 CrossRef Medline

7. Alsop DC, Detre JA. Reduced transit-time sensitivity in noninvasive magnetic resonance imaging of human cerebral blood flow. J Cereb Blood Flow Metab 1996;16:1236-49 CrossRef Medline

8. MacIntosh BJ, Filippini N, Chappell MA, et al. Assessment of arterial arrival times derived from multiple inversion time pulsed arterial spin labeling MRI. Magn Reson Med 2010;63:641-47 CrossRef Medline
9. Haller S, Zaharchuk G, Thomas DL, et al. Arterial spin-labeling perfusion of the brain: emerging clinical applications. Radiology 2016; 281:337-56 CrossRef

10. Alsop DC, Detre JA, Golay X, et al. Recommended implementation of arterial spin-labeled perfusion MRI for clinical applications: a consensus of the ISMRM perfusion study group and the European consortium for ASL in dementia. Magn Reson Med 2015;73:102-16 CrossRef Medline

11. Wells JA, Lythgoe MF, Gadian DG, et al. In vivo Hadamard encoded continuous arterial spin labeling (H-CASL). Magn Reson Med 2010; 63:1111-18 CrossRef Medline

12. Wang DJ, Alger JR, Qiao JX, et al; UCLA Stroke Investigators. Multidelay multi-parametric arterial spin-labeled perfusion MRI in acute ischemic stroke: comparison with dynamic susceptibility contrast enhanced perfusion imaging. Neuroimage Clin 2013;3:1-7 CrossRef Medline

13. Haller S, Montadon ML, Rodriguez C, et al. $\mathrm{APOE}^{\star} 4$ is associated with gray matter loss in the posterior cingulate cortex in healthy elderly controls subsequently developing subtle cognitive decline. AJNR Am J Neuroradiol 2017;38:1335-42 CrossRef Medline

14. Dai W, Robson PM, Shankaranarayanan A, et al. Reduced resolution transit delay prescan for quantitative continuous arterial spin labeling perfusion imaging. Magn Reson Med 2012;67: 1252-65 CrossRef Medline

15. Smith SM, Nichols TE. Threshold-free cluster enhancement: addressing problems of smoothing, threshold dependence and localisation in cluster inference. Neuroimage 2009;44:83-98 CrossRef Medline

16. Liu Y, Zhu X, Feinberg D, et al. Arterial spin labeling MRI study of age and gender effects on brain perfusion hemodynamics. Magn Reson Med 2012;68:912-22 CrossRef Medline

17. Seghier M. Laterality index in functional MRI: methodological issues. Magn Reson Imaging 2008;26:594-601 CrossRef Medline

18. Liebeskind DS. Collateral circulation. Stroke 2003;34:2279-84 CrossRef Medline

19. Türe U, Yaşargil MG, Al-Mefty O, et al. Arteries of the insula. J Neurosurg 2000;92:676-87 CrossRef Medline

20. Guo J, Lebel M, Holdsworth S, et al. Hadamard-encoded multi-delay PCASL: should the bolus durations be T1-Hadamard-encoded adjusted? In: Proceedings of the Annual Meeting and Exhibition of the International Society for Magnetic Resonance in Medicine, Honolulu, Hawaii. April 22-27, 2017 